\title{
ADRIANA AMARAL
}

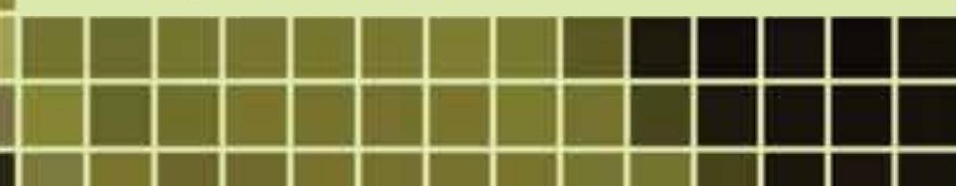


ADRIANA AMARAL

é professora

do Mestrado e

Doutorado em

Comunicação e

Linguagens da

Universidade Tuiuti

do Paraná.

\section{Etnografia e}

\section{pesquisa}

\section{em Gibereultura:}

\section{limites e}

\section{insufieiêneias}

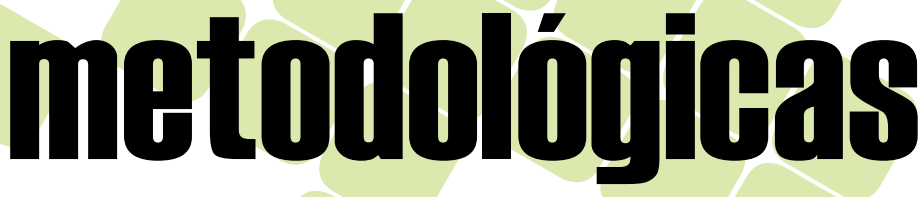




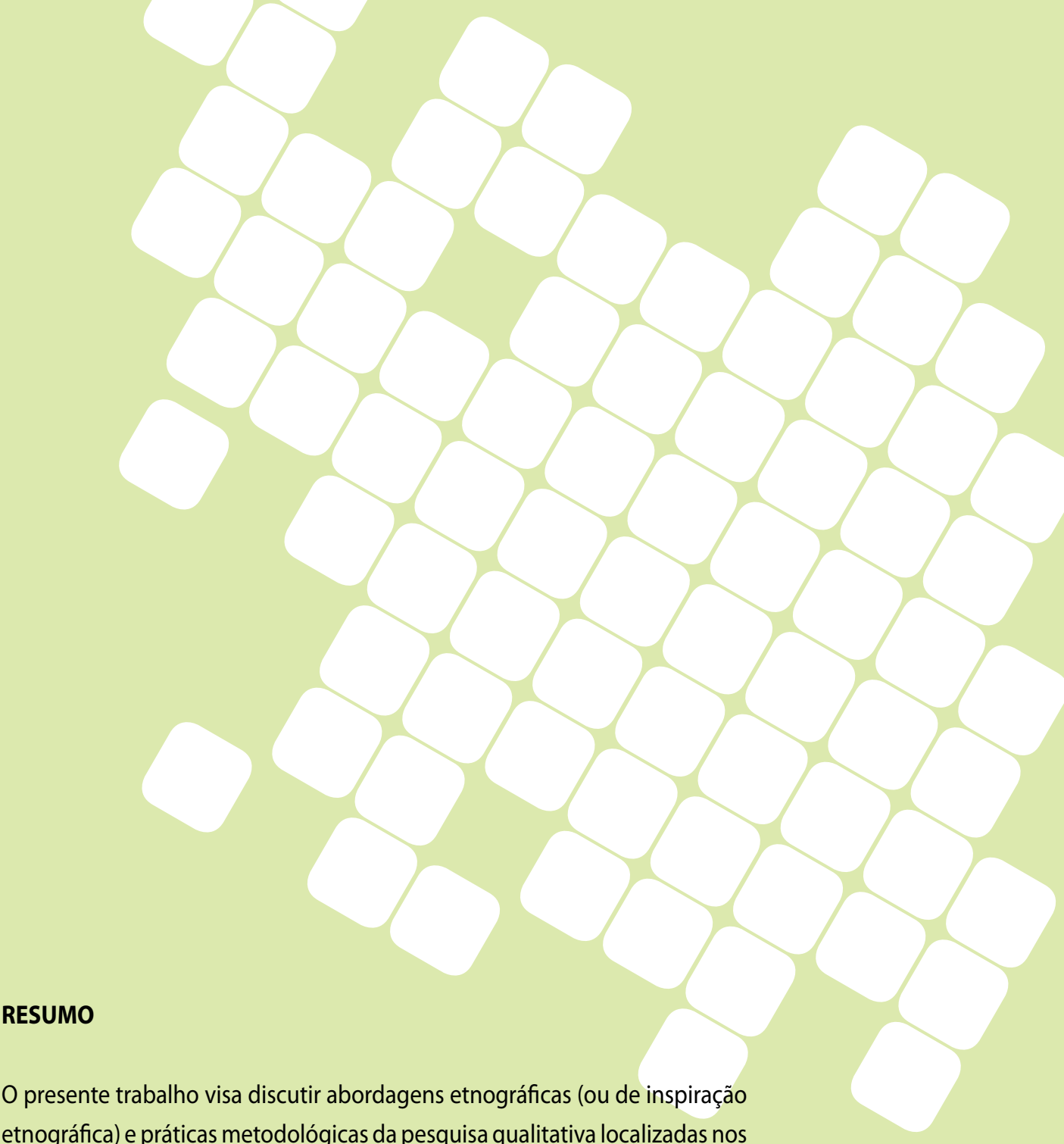
etnográfica) e práticas metodológicas da pesquisa qualitativa localizadas nos estudos empíricos sobre cibercultura, com ênfase nas culturas, práticas comunicacionais e relações sociais emergentes. Tais aproximações são observadas em sua complexidade e limitações, a partir de experiências de pesquisa e no design da construção do campo (Markham \& Baym, 2009).

Palavras-chave: metodologia, etnografia, cibercultura.

\begin{abstract}
This paper aims at discussing ethnographical (or ethnographically-inspired) approaches on empirical studies on cyberculture, and methodological practices of qualitative research focused on cultures, and emerging social networks and communicational practices. Such ethnographical approaches are analyzed in their complexities and limitations, from the viewpoint of research experiences and also on the field design and construction (Markham \& Baym, 2009).
\end{abstract}

Keywords: methodology, ethnography, cyberculture. 


\section{INTRODUÇÃOO}

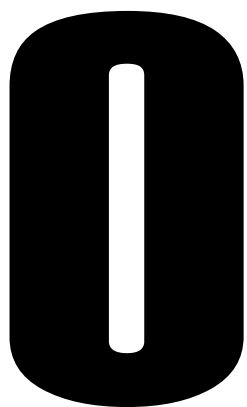

presente artigo rediscute abor-

dagens a respeito dos usos da etnografia (ou estudos de inspiração etnográfica) como uma das metodologias utilizadas no estudo empírico da Internet, partindo de um modelo comunicacional que leve em conta seu contexto e as culturas que nela se desenvolvem, na qual estão inscritos tracejados e conversações cuja observação sistemática e investigação interpretativa desvendam padrões de comportamento social e cultural.

Num primeiro momento questionaremos algumas terminologias utilizadas para descrever as apropriações da metodologia. Depois, trataremos de alguns de seus princípios e etapas de desenvolvimento, como a entrada em campo, a análise e métodos complementares, as relações entre pesquisador e informantes e a questão da privacidade e da ética de pesquisa. Além de discutir alguns procedimentos, apontaremos exemplos retirados de experiências com a pesquisa de cunho qualitativo nos quais a diversidade de objetos proporcionadoa pela cibercultura.

\section{MAPEANDOTERMINOLOGIAS}

Desde o estabelecimento da Internet como meio de comunicação e da "constituição" de grupos sociais possibilitados pelas facilidades da comunicação em rede, alguns pesquisadores perceberam que as técnicas de pesquisa etnográficas também poderiam ser utilizadas para o estudo das culturas e comunidades agregadas via Internet, fossem elas derivadas de grupos sociais já constituídos no offline e que, neste momento, migram e/ou transitam entre esses espaços, ou mesmo de formações sociais compostas apenas por relações online.

A transposição do método a partir dos anos 90 gerou inúmeros debates. Muitos antropólogos e cientistas sociais mais ortodoxos não aceitaram a proposição (Kozinets, 2010), principalmente em função de uma reconfiguração em algumas das principais características da etnografia e do fazer etnográfico. Para esses autores, o deslocamento, o estranhamento e o "ir a campo",tão decisivos na formação do olhar interpretativo, pareciam ter se esvaído frente a uma reconfiguração espaço-temporal advinda das TICs. Assim há transformações diretas no fazer etnográfico.

Para Hine (2000, 2005), responsável pela popularização do termo, na etnografia virtual a construção do campo se dá a partir da reflexividade e da subjetividade (Hine, 2009). Segundo a autora, a etnografia virtual se dá no e através do online e nunca está desvinculada do offline, acontecendo por meio da imersãoe engajamento intermitente do pesquisador com o próprio meio.

Outra variação foi a criação do neologismo "netnografia" (net + etnografia), cunhado na metade dos anos 90 . Kozinets popularizou o termo em suas pesquisas relacionadas ao fandoma, ao marketing e às comunidades de consumo online. No contexto nacional, o artigo de Sá (2002) sobre netnografia deu início a um ainda lento debate sobre as repercussões dessa metodologia, ampliado por outros autores como Montardo e Rocha (2005), Amaral, Natal e Viana (2008), entre outros.

Kozinets (2010, p. 4) defende o uso do termo, relembrando que a própria palavra "etnografia" é um neologismo cunhado no início do século XIX. O autor também acredita que a utilização desse termo demarca e pontua as diferenças que o método etnográfico sofre quando adaptado para os ambientes digitais seja em termos de forma de coleta de dados, seja em termos de ética de pesquisa e análise, uma vez que o presencial e as experiências são de naturezas diferenciadas. Ele enfatiza a inter-relação entre "vida social online e os mundos sociais da "vida real"' (Kozinets, 2010, p. 2).

$\mathrm{O}$ intenso relacionamento entre as aplicações da netnografia voltadas aos estudos no campo do marketing e do consumo no contexto das redes e a análise de potenciais 
consumidores e seus comportamentos é que possibilitam a vinculação do termo às pesquisas aplicadas de mercado. Para Montardo e Rocha (2005, p. 13), "nota-se que muitos sites descrevem netnografia como o monitoramento de comunidades online a fim de se estabelecer hábitos de consumo".

Essa observação demonstra que há, em geral, uma falta de compreensão em relação a sua complexidade teórico-epistemológica, ao limitá-la a um mero instrumento de aferimento de audiência e consumo, descartando o entendimento das práticas comunicacionais num sentido mais amplo, e dos aspectos protocolares culturais e comportamentais de cada grupo.

\section{Outras terminologias}

Do flerte entre as pesquisas de mercadoe as pesquisas acadêmicas criou-se uma gama de outros termos (ou seriam meramente definições operatórias?) como "etnografia digital" e "webnografia", por exemplo. Alguns autores criticam os neologismos e afirmam que eles caberiam sob o guardachuva da ciberantropologia. Tais terminologias não propõem mudanças substanciais à etnografia, mas em relação à maneira de lidar com os diferentes procedimentos e tipos de suporte, não parecendo incorporar uma reflexão mais profunda sobre a sua materialidade, como podemos observar nessa breve sistematização (Tabela 1).

Kozinets (2010, p. 6) questiona a “confusão epistemológica" e política - no âmbito das publicações acadêmicas - gerada por esses múltiplos termos, afirmando que essas distinções não são necessárias e tendem a gerar padrões diferentes que dificultam avaliações dos resultados obtidos pelos pares.

Hine (2009) também questionou o surgimento de tantos termos, propondo uma suplantação da “etnografia virtual”, uma vez que o termo alude a uma suposta distinção entre os ambientes online e offline em vez de uma relação de contiguidade. Para a autora, estaríamos em uma fase distinta de quando ela escreveu o livro Virtual Ethnography
(2000), questionando se "has 'virtual ethnography' simply collapsed back into 'ethnography"'.

A questão nos coloca frente a uma tomada de posicionamento que incide sobre o desenho e o planejamento dos métodos de pesquisa, ao que ela recomenda um cuidado, pois há diferenças entre online e offline que devem ser mantidas nas descrições das práticas de pesquisa. Por exemplo, a diferença entre uma entrevista feita presencialmente e outra feita via MSN ou Skype deve ser incluída na narrativa. O refinamento das análises sofrerá influências que podem ser significativas.

\section{O DESIGNDA PESQUISAE A CONSTRUÇÃO DO CAMPO}

Em 2000, Hine propôs que a Internet enquanto objeto de estudo tende a ser elaborada conceitualmente sob dois modelos de abordagem teórica: Internet enquanto cultura e enquanto artefato cultural.

Cultura-Enfoca o contexto cultural dos fenômenos que ocorrem nas comunidades e/ou mundos virtuais. Essa abordagem leva em consideração funções, formações sociais, tipos de organizações, conflitos e cooperações, como nos textos de Rheingold (1993) e Jones (1999). Os estudos sobre experimentações das identidades online também aparecem em diversos trabalhos considerados já clássicos, como o de Stone (1991), que analisa a questão do corpo, e de Turkle (1997), que debate a multiplicidadee os jogos identitários dos usuários de MUDs.

Artefato cultural - Observa a inserção da tecnologia na vida cotidiana e compreende que existem diferentes significados culturais em contextos diferentes. O objeto Internet não é único, mas sim multifacetado e passível de apropriações.

Os estudos cujas abordagens enfatizam o aspecto de artefato cultural tendem a analisar os discursos sobre a história e as 


\section{Tabela 1}

\section{OUTRAS TERMINOLOGIAS}

\begin{tabular}{|c|c|c|c|}
\hline & Etnografia digital & Webnografia & Ciberantropologia \\
\hline $\begin{array}{c}\text { Definiçõos e tipo de } \\
\text { pesquisa }\end{array}$ & $\begin{array}{l}\text { Explorar e expandir } \\
\text { as possibilidades da } \\
\text { etnografia virtual através } \\
\text { do constante uso das } \\
\text { redes digitais, postando o } \\
\text { material coletado. Criação } \\
\text { de narrativas audiovisuais } \\
\text { colaborativas em uma } \\
\text { linguagem que sirva como } \\
\text { material de estudo e } \\
\text { também atinja um público } \\
\text { não acadêmico. }\end{array}$ & $\begin{array}{l}\text { Alguns autores o utilizam } \\
\text { relacionado à pesquisa } \\
\text { aplicada de marketing } \\
\text { na Internet, enfatizando } \\
\text { as métricas e audiências, } \\
\text { principalmente em } \\
\text { ambientes de discussão. } \\
\text { Para Dann e Forrest (1999), } \\
\text { "Webnography describes the } \\
\text { combination of techniques } \\
\text { associated with content } \\
\text { analysis, and ethnographic } \\
\text { research to analyze 'interest } \\
\text { clusters' that have formed in } \\
\text { the USENET and Webthread } \\
\text { environments". } \\
\text { Outros compreendem o } \\
\text { termo como o método não } \\
\text { restrito a etnógrafos, mas } \\
\text { aberto a pesquisadores } \\
\text { interessados nos } \\
\text { complexos aspectos } \\
\text { sociais, culturais e } \\
\text { psicológicos relacionados } \\
\text { com e através da Internet. } \\
\text { (Ryan, 2008). }\end{array}$ & $\begin{array}{l}\text { Estudo dos humanos nos } \\
\text { ambientes conectados. } \\
\text { Baseia-se nos conceitos } \\
\text { da antropologia ciborgue } \\
\text { de Haraway (1991) para } \\
\text { examinar a reconstrução } \\
\text { tecnológica do homem e } \\
\text { preparar o etnógrafo para } \\
\text { lidar com uma categoria } \\
\text { mais ampla de "ser } \\
\text { humano". }\end{array}$ \\
\hline Pesquisadores & $\begin{array}{l}\text { Grupo da Kansas State } \\
\text { University coordenado por } \\
\text { Michael Wesch }\end{array}$ & $\begin{array}{l}\text { Segundo a } \\
\text { "Webnographers Wiki", wiki } \\
\text { mantida por pesquisadores } \\
\text { norte-americanos, projetos } \\
\text { como o Digital Youth }{ }^{2} \text { e } \\
\text { The Facebook Project }{ }^{3} \text {, etc. } \\
\text { podem ser enquadrados } \\
\text { nesse tipo de proposta. }\end{array}$ & $\begin{array}{l}\text { Donna Haraway (1991, } \\
\text { 1996), Arturo Escobar } \\
\text { (1994) e David Hakken } \\
\text { (1999) são referências } \\
\text { fundadoras, no entanto, } \\
\text { alguns autores, como John } \\
\text { Postill, defendem o uso do } \\
\text { substituto à "netnografia" } \\
\text { ou "etnografia virtual". }\end{array}$ \\
\hline Sites de referência & $\begin{array}{l}\text { http://mediatedcultures. } \\
\text { net/ksudigg/ }\end{array}$ & $\begin{array}{l}\text { http://www. } \\
\text { webnographers.org/index. } \\
\text { php?title=Main_Page }\end{array}$ & \\
\hline Outras observações & $\begin{array}{l}\text { O grupo popularizou suas } \\
\text { ações através do premiado } \\
\text { vídeo The Machine Is Us/Ing } \\
U s^{4} \text { postado no YouTube. }\end{array}$ & $\begin{array}{l}\text { Mescla conceitual } \\
\text { entre webnografia } \\
\text { e ciberantrologia } \\
\text { ao considerarmos } \\
\text { as descrições da } \\
\text { "Webnographers Wiki". } \\
\text { Nessa concepção, o termo } \\
\text { pode servir tanto para } \\
\text { pesquisas acadêmicas } \\
\text { quanto mercadológicas. }\end{array}$ & \\
\hline
\end{tabular}


concepções da Internet, como, por exemplo, os discursos libertários acerca da natureza anárquica e da atitude contracultural dos hackers e cyberpunks do início da rede, como em Turner (2006), entre outros, além dos processos de produção e consumo na construção do sentido dos seus usos, questionando os processos comunicacionais em relação aos papéis do emissor e do receptor.

As práticas de produção e consumo de conteúdo através dos usuários acabam oportunizandoum amplo recorte de análise. Nessa abordagem, o papel das audiências pode ser enfatizado e relativizado em seus aspectos positivos e negativos, a partir de uma intrínseca relação com os estudos culturais, como propõe Sterne (1999), sob a noção antevista por Raymond Williams (1974), de que as tecnologias são sempre produzidas por um processo histórico e em um sistema social.

Um exemplo sobre esse tipo de definição está na análise etnográfica da relação entre plataformas de música online e gêneros musicais encontrada em Amaral (2007), na qual foi elaborado um levantamento dos sites de redes sociais de música mais utilizados pelos usuários em geral a partir de um ranking observado pela audiência através da ferramenta Alexa ${ }^{5}$ e das matérias dos blogs relevantes em termos de tecnologia, como "ReadWriteWeb", "TechCrunch" e "Mashable". Assim, foram considerados como campo, num primeiro mapeamento, Last.fm, Pandora e Myspace. Quando levantada a hipótese da folksonomia e da categorização de gêneros através das tags, $\mathrm{o}$ Myspace foi descartado por não ter sistema de tagueamento, e Pandora foi desconsiderado por não estar acessível para usuários de fora dos Estados Unidos, restando assim a análise do Last.fm.

\section{Observação e processo}

\section{interpretativo no campo}

Segundo Kendall (2009), o começo e cidade de ferramentas e métodos complementares utilizados, e do contexto cultural. A definição do objeto emerge a partir dos questionamentos a ele endereçados e não a partir de predeterminações. Ainda de acordo com a autora, devem ser consideradas três fronteiras (espacial, temporal e relacional) e três esferas de influência (analítica, ética e pessoal) para a construção do campo a ser pesquisado.

"Spatial boundaries refer to questions of where, who, and what to study. Temporal boundaries refer to questions of time spent and the issues of beggining and ending the research. Relational boundaries refer primarily to relationships between researchers and the people they study (although other relationships are also pertinent to research projects, such as relationships between researchers and their audience for written reports). The analytical sphere of influence refers to theoretical and analytical decisions regarding project boundaries. The ethical sphere of influence refers to boundary decisions madefor ethical reasons, especially those made to protect participants. The personal sphere of influence refers to various aspects of the researcher's background that might influence the choice of project boundaries, such as personal proclivities, skills or history" (Kendall, 2009, p. 22).

Para Winkin (1998, p. 132), o processo de construção da etnografia consiste em saber ver, saber estar com e saber escrever. Os atos descritivos incluem uma série de protocolos a serem devidamente organizados, recomendando-se alguns cuidados como:

a) Entrar em contato com o grupo - Essa primeira fase de ambientação é o que Kozinets (2002) chama de entrée cultural e o que o circunda, examinando atentamente sua infraestrutura social e técnica. Winkin (1998, p. 142) aponta as dificuldades da entrada em campo, como a negociação com os informantes, a procrastinação e uma certa cegueira que atinge o pesquisador nos primeiros instantes da observação, nos quais ele parece "não ver nada"; 
b) Manter um diário de campo com as anotações - Por calcar-se na observação, o método etnográfico dá especial atenção à utilização do chamado "diário de campo", onde serão anotadas todas as impressões do pesquisador sobre o cotidiano dos pesquisados. Independente do suporte (um caderno, folhas, computador, gravadores), essas anotações são fundamentais para o momento final da produção da etnografia. Winkin (1998, p. 139) comenta a existência de três funções para o diário de campo: emotiva, empírica e reflexiva e analítica. No caso da etnografia feita nos meios digitais, aumentase a possibilidade do uso de ferramentas como um blog não apenas como objeto de pesquisa, mas como o próprio diário de campo e ferramenta de coleta de dados a partir dofeedback com os participantes e da interatividade que lhe são característicos, conforme problematizam alguns autores como Ward (2006) e Amaral (2009);

c) Contextualizar os informantes e usar diversos tipos de entrevistas - É importante enfatizar que nem só de observação vive a pesquisa de campo, mas também de intercâmbios e trocas culturais e de ouvir os informantes.

A vigilância e a constante organização dos procedimentos de campo não visam a confundir a etapa de observação participante com a etnografia em si, desvio facilmente manipulável quando se trata das facilidades da observação e da coleta de dados na Internet, e que pode refletir em falta de análise qualitativa dos dados e de relatos mais densos, comprometendo-se em rotular-se como etnografia quando se mantém somente nas etapas observacionais e descritivas, entrando de forma incipiente nas entrevistas, na análise e reflexão dos dados coletados via sites de redes sociais, blogs, etc. A etnografia torna-se, assim, um sinônimo supostamente legitimado para uma mera observação e monitoramento, talvez por ter sido interpretada de uma forma simplista por conta da disseminação dos "usos" do termo pela pesquisa de mercado e na própria rede, conforme apontamos anteriormente.

\section{Ampliando o espectro metodológico e os objetos possíveis}

Por conta dessas ampliações, mudanças e complementações nos procedimentos metodológicos empíricos e das várias disciplinas que estudam a Internet é que autores como Denzin (2004) e Markham e Baym (2009) optam por um termo mais amplo: "pesquisa qualitativa".

"In common definition and traditional application, qualitative methods have been associated with close analysis and interpretation by the researcher, trained in various specific methods of information collection (e.g, interviewing, participantobservation in the field, notation or collection of such things as written texts, transcripts of conversations, documents, andartifacts) and even in more specific methods of data analysis within one's school of thought (e.g., conversation analysis, grounded theory, deconstruction, rhetoricalcriticism, network analysis, phenomenology, and so forth)" (Markham \& Baym, 2009, p. xii).

A combinação multimétodos reforça e desvela o caráter epistêmico da etnografia e está presente em estudos que priorizam objetos distintos da cibercultura. Muitos exemplos e relatos podem ser observados em coletâneas específicas sobre o assunto, como Jones (1999), Johns, Chen e Hall (2004), Hine (2005) e Markham e Baym (2009), etc. Tais combinações operam ora em níveis macro, ora em micro, ora em mezzo.

"The macro level, which deals mainly with epistemological issues and which often finds grounding in one of the three main approaches to social science research: positivism, interpretative investigation, and critical analytical studies. The mezzo level, which is mainly concern with issues regarding research design and strategy, such as employment of multiple methods, development 
of longitudinal studies and consideration of different theoretical and disciplinary approaches. The micro level, which is concerned with specific methods and techniques and includes such topics as gathering log data and conducting web surveys" (Jankowski \& Van Selm, 2005, pp. 201-2).

Em nível macro, há a amplificação do espectro teórico,proporcionando discussões interdisciplinares e epistemológicas sobre o campo, refletida em aspectos conceituais distintos como a investigação interpretativa de tradição abertamente antropológica, os estudos culturais, as materialidades, o interacionismo simbólico, a teoria crítica, entre outros. Em termos médios, a própria elaboração do design da pesquisa de campo e as estratégias de construção do objeto tendem a inferir nas escolhas teóricas e metodológicas e nos possíveis avanços da pesquisa. Já em relação ao nível micro, a preocupação centra-se na especificidade dos métodos e técnicas específicas em relação aos possíveis objetos da pesquisa em Internet e no compartilhamento de experiências acerca daquele determinado objeto, conforme podemos observar nos exemplos a seguir.

"The netnographic approach is adapted to help the researcher study not only forums, chat, and newsgroups butalsoblogs, audiovisual, photographic, and podcasting communities, virtual worlds, networked game players, mobile communities, and social networking sites" (Kozinets, 2010, p. 3).

Montardo (2009) conjuga a netnografia à análise de redes sociais em seus estudos sobre inclusão social em redes temáticas na Web. Duarte (2008) parte da premissa teórica das subculturas e pós-subculturas como formas culturais disseminadas nas redes sociais e que podem ser observadas a partir da etnografia em seus protocolos de comunicação e práticas sociais das comunidades de cosplayers no Orkut. Gutierrez (2009) indica a questão da dialética materialista como forma de abordagem da etnografia de redes sociais no campo da educomunicação tendo como objeto os blogs de professores do ensino médio.

Natal (2009) propõe a articulação da netnografia com a análise de conversação para a observação da constituição de uma "lovemark" online seja através do blog ou do perfil da marca Mary Jane no Orkut . Amaral (2007) utiliza websurveys, observação sistemática dos perfis, entrevistas informais através de comunicadores instantâneos (MSN, Gtalk) e inserções em eventos offline para interpretação do material textual relativos à categorização de gêneros musicais pela subcultura industrial na plataforma Last.fm. Strangelove (2007) utilizou gravações em vídeo dos jogadores do game Battlefield 2 para análise e interpretação dos significados da violência. Ruby (2009) defende a importância dos meios interativos e multimídia como alternativa aos tradicionais filmes etnográficos, propondo a criação de blogs, sites, perfis e outros, como alternativa. Já Mitsuishi (2007) tece críticas e analisa as abordagens etnográficas nos estudos em games e mundos virtuais. Assim, são inúmeras as possibilidades de objetos que podem ser recortados no campo das redes digitais, sejam eles exclusivamente online ou híbridos (online e offline).

\section{Graus de inserção do pesquisador e questões éticas}

Outro apontamento importante de Hine (2009) trata do engajamento e da biografia do pesquisador como item essencial para uma discussão metodológica. A autora reflete sobre sua própria trajetória como pesquisadora como ponto de partida para a temática de sua mais recente pesquisa, além da sua relação imersiva como usuária experiente de documentos e bases de dados taxonômicas sobre ciências desde o final dos anos 80. Também o relato das dificuldades e habilidades do pesquisador em relação à utilização das ferramentas digitais (Markham, 1998) elencadas para o recorte da análise no campo redimensiona o seu papel subjetivo e proporciona debates 
sobre a experimentação das ferramentas e a confiabilidade dos dados coletados.

Evidentemente que a partir da inserção do pesquisador no campo, mesmo que ele não se identifique e não seja um participante previamente inserido na cultura em questão, há uma transformação no objeto. Além disso, a decisão da permanência ou nãoem silêncio (Hine, 2005), ou através das práticas de lurking ${ }^{6}$ (Orgadi, 2009), precisa ser tomada e refletida, influenciando assim as escolhas, justificativas e direcionamentos éticos que acontecerão ao longo da pesquisa e que terão reflexos em seus resultados.

Duarte (2008), Orgadi (2009), Montardo (2009) e Natal (2009) optam pela observação "silenciosa" nas culturas devido a questões de ordem ética e privacidade em relação aos informantes quanto a faixa etária,prejulgamentos ou material/conteúdo sensível (como doenças, representações midiáticas dos grupos, etc.).

Assim como o pesquisador observador silencioso ou lurker implica limitações e benefícios para os resultados da pesquisa, o chamado insider (Hodkinson, 2005) também compromete a narrativa etnográfica,

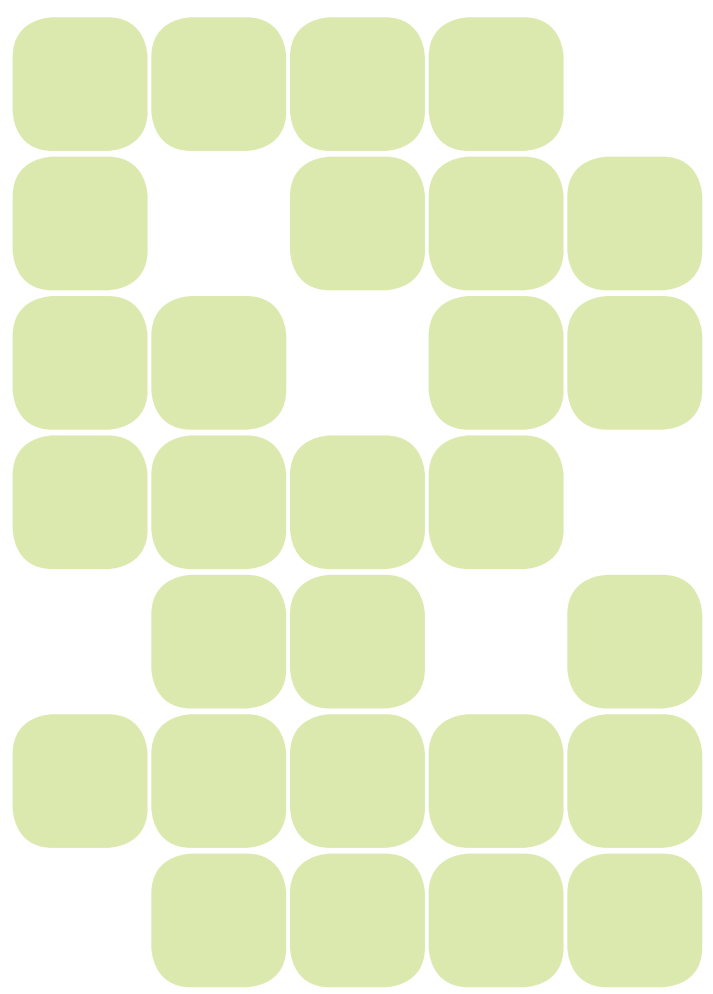

com a inserção de elementos autobiográficos e seu pré-conhecimento e/ou participação da cultura observada. Segundo Hodkinson (2005), estudioso das culturas juvenis underground offline e online, ser insider constitui um "conceito não absoluto intencionado para designar aquelas situações caracterizadas por um grau significante de proximidade inicial entre as locações socioculturais do pesquisador e do pesquisado", ou o que Jenkins (2006) chama em suas pesquisas sobre os fandoms de ACA-Fan ("acadêmico-fã" ou "pesquisador-fã"). Em tais casos faz-se necessário destacar e problematizar tal posição autonetnográfica como fazem Hodkinson (2005), Brill (2008), Viana (2009) e Amaral (2009), por exemplo, menos por uma justificativa ou facilidade pela escolha do recorte e/ou comunidade, subcultura ou grupo, do que pela cautela ao interpretar dubiamente o material, ou em perceber os relatos, ou mesmo em exacerbar a representatividade de um universo experiencial.

Atenta às problemáticas do insider, Brill (2008), em sua pesquisa sobre a subcultura gótica e suas representações na mídia (offline e online), expõe sua estratégia ao lidar com a carga simbólica, subjetiva e ética do nível insider: "I more and more developed the skill of using differing degrees of 'distance' and 'closeness' strategically, managing to frame my field experiences accordingly" (Brill, 2008, p. 2)

Uma das principais dificuldades na condução dos tópicos de ética relativos à Internet diz respeito às noções de público e privado e, por consequência, em decidir o que é ou não público e, portanto, passível de divulgação nos resultados da pesquisa (Elm, 2009) ou mesmo no feedback com os informantes. Segundo Jones (2004, p. 184), esse tipo de decisão levanta discussões sobre diferentes tipos de sensibilidades que devem servir como "linhas guias em nossos esforços para estudar as culturas emergentes".

Para Ess (2009), a privacidade é uma questão de cunho cultural e geracional, compreendida e valorizada sob diferentes modos em diferentes sociedades. Elm
6 Ato de entrar em listas de discussão, fóruns, comunidades online, etc. apenas como observador, sem participação ativa. 
(2009) acredita que, entre outras coisas, ela diz respeito à integridade individual e ao direito à autodeterminação, implicando, assim, em diferentes níveis do que é considerado público e privado quando da análise das culturas emergentes na Web ou híbridas (entendendo por híbrido atuações e laços indistintos entre online e offline).

$\mathrm{Na}$ análise dos ambientes online, Elm (2009, p. 75) também estipula quatro níveis de privacidade a serem observados: 1) público - aberto e disponível a todos; 2) semipúblico-disponível a quase todos. Requer ser membro e/ou ter cadastro; 3 ) semiprivado - requer pertencer à organização de forma mais profunda; 4) privado - indisponível e fechado.

Tais níveis são bastante discutíveis e questionáveis devido a sobreposições e conflitos entre eles, contudo a autora afirma que devem ser gradualmente alterados e levando em consideração se o ambiente escolhido e as pessoas são públicos o suficiente para serem estudados sem ter o consentimento dos informantes.

A autora também salienta que a análise do que é público e privado depende da percepção do pesquisador (seja seu lugar de fala, identidade, cultura, etc.), não se tratando de um fato a priori. Uma questão que não deve ser desconsiderada nessa reflexão é relativa à audiência, ou seja, "para quem o conteúdo é criado ou para qual audiência ele é dirigido" (Elm, 2009, p. 77). A partir de tais respostas é possível desvelar se a natureza do conteúdo é considerada como material sensível e, assim, “desenhar” as possíveis opções éticas tais como método de acesso, identificação do pesquisador, temáticas escolhidas, preservação dos nomes dos entrevistados sob pseudônimos ou identificação.

O material sensível abrange uma gama de critérios como a faixa etária dos participantes da cultura, suas profissões, temáticas debatidas no ambiente, etc. Exemplos: as redes temáticas de inclusão digital, como redes de usuários e/ou pais com autismo, síndromes de Asperger e de Down pesquisadas por Montardo (2009), assim como as conversações no blog da marca Mary Jane, analisadas por Natal (2009), que possuíam um caráter de debate psicológico como pedidos de conselhos sobre necessidades especiais - no caso de Montardo-e sexuais - no caso de Natal - entre meninas adolescentes que postavam os comentários, e por isso foram consideradas material sensível, tendo seus nomes verdadeiros omitidos do relatório final da dissertação.

De maneira oposta, os perfis do Myspace e Last.fm, analisados por Amaral (2007), que foram expressamente autorizados pelos próprios membros da subcultura eletroindustrial que fizeram questão de que seus nicks e/ou nomes verdadeiros fossem divulgados nos resultados da pesquisa, uma vez que as postagens e informações neles contidos tratavam de construção de identidades a partir de gêneros musicais, não foram considerados conteúdos sensíveis.

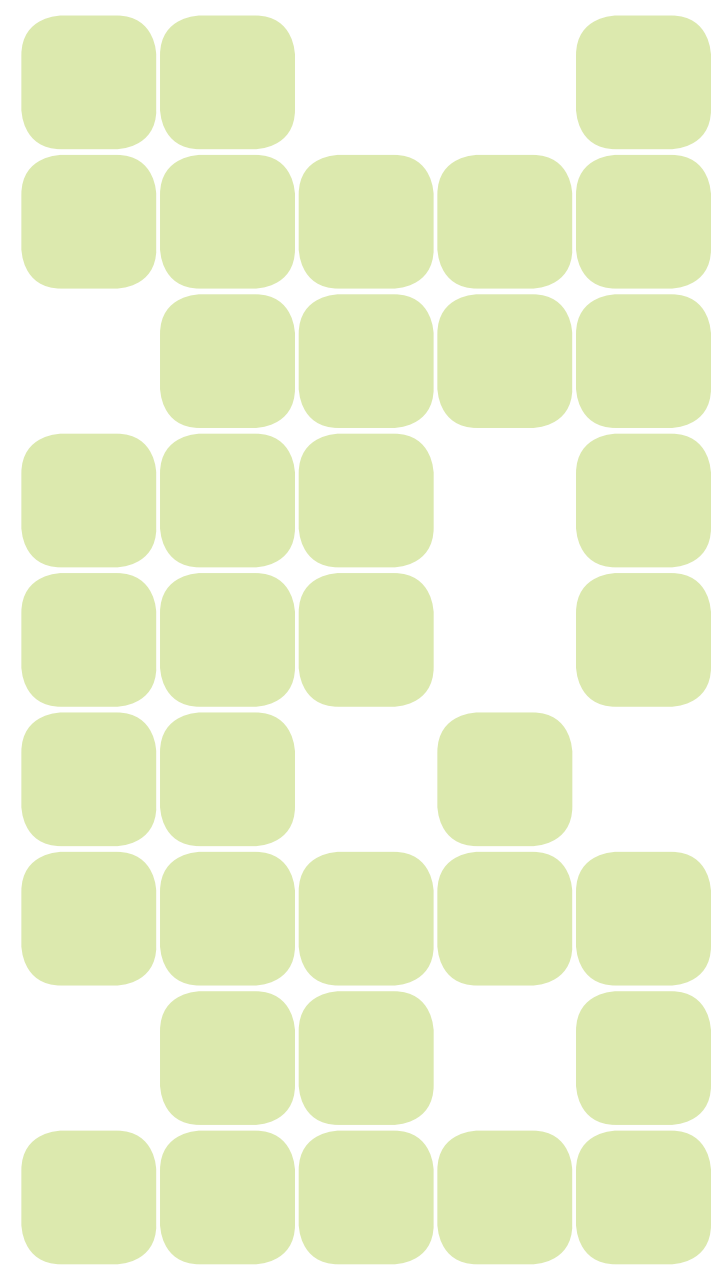




\section{CONSIDERAÇÕES FINAIS}

Neste artigo, discutimos algumas abordagens etnográficas da pesquisa em cibercultura com ênfase na observação e análise das culturas emergentes, sejam elas exclusivamente online ou híbridas. Procuramos abordar as apropriações e procedimentos metodológicos que a etnografia vem sofrendo ao ser transposta para as mídias digitais, em especial a Internet. Problematizamos as diferentes terminologias, muitas delas derivadas das aplicações mercadológicas e suas definições, que funcionam ora como instrumentos de operacionalização, em nível de protocolos metodológicos em um contexto micro ou mezzo (Jankowski \& Van Selm, 2005), ora como conceituações plurais, que procuram teorizar o macro, nos remetendo a questões mais amplas de ordem epistemológica, na qual "o estudo do ordinário é feito com base nos dados 'extraordinários"” (Winkin, 1998, p. 160).

Procuramos decompor alguns princípios da construção do campo, a partir do compartilhamento de experiências, relatos e exemplos retirados de pesquisas de cunho etnográfico multimétodos, tendo como pressuposto as múltiplas narrativas etnográficas possibilitadas pelos processos de interpretação da comunicação nos ambientes online, independentemente das fronteiras e limitações espaço-temporais.

Ainda há muito a ser problematizado em torno das abordagens etnográficas dos estudos sobre Internet e outras tecnologias de comunicação - assim como discussões sobre outras metodologias de ordem empírica e teórica - para a compreensão da cultura digital que se esparrama pelo offline através de aplicativos, ferramentas de realidade aumentada, comentários e conversações em microblogs e novos formatos e produtos comunicacionais que, ao surgirem, carregam tracejados simbólicos, códigos e outros padrões de comportamento cultural inscritos a partir da sociedade que os desenvolveu.

Esperamos que nossos apontamentos, calcados tanto no levantamento bibliográfico quanto nas experiências empíricas de pesquisas desenvolvidas, possam contribuir para a ampliação do debate dos estudos sobre Internet e cibercultura, em especial no contexto brasileiro, já notoriamente reconhecido pelas reflexões em nível teórico, mas ainda carente de bibliografia em língua portuguesa sobre discussões e formulações de propostas metodológicas essenciais para o avanço da área.

\section{BIBLIOGRAFIA}

AMARAL, A. "Categorização dos Gêneros Musicais na Internet - Para uma Etnografia Virtual das Práticas Comunicacionais na Plataforma Social Last.fm", in J. Freire Filho; M. Herschmann (orgs.). Novos Rumos da Cultura da Mídia. Indústrias, Produtos e Audiências. Rio de Janeiro, Mauad, 2007, pp. 227-42.

AMARAL, A. "Autonetnografia e Inserção Online: o Papel do Pesquisador- Insider nas Práticas Comunicacionais das Subculturas da Web", in Revista Fronteiras - Estudos Midiáticos, v. 11, 2009, p. 14-24. Disponível em: http://www.fronteiras.unisinos. br/pdf/62.pdf . Acesso: 5/12/2009.

AMARAL, A.; NATAL, G.; VIANA, L. "Netnografia como Aporte Metodológico da Pesquisa em Comunicação Digital", in Revista Sessões do Imaginário, Porto Alegre, v. 2, n. 20, dez./2008, pp.34-40. Disponível em: http://revistaseletronicas.pucrs.br/ojs/index.php/famecos/article/viewFile/4829/3687. Acesso: 20/1/2010. 
ARDÈVOL, E. et al. "Media Practices and the Internet: Some Reflections Through Ethnography", in Presentación en el Simposio del XI Congreso de Antropología de la FAAEE. Donostia, 10-13 de septiembre de 2008. Disponível em: http://www.slideshare.net/Estalella/ towards-an-ethnography-of-new-media-practices-reflections-through-field-experiencepresentation?src=embed. Acesso: 1/2/2010.

BRILL, D. Goth Culture. Gender, Sexuality and Style. New York, Berg, 2008.

DANN, S.; FORREST, E. Webnography. Developing Unobstrusive Online Research. Disponível em: http://www.cbpp.uaa.alaska.edu /afef/webnography.htm. Acesso: 19/8/2009.

DENZIN, N. K. "Prologue: Online Environments and Interpretative Social Research", in Mark D. Johns; Shing-Ling S. Chen; G. Hall. Online Social Research. Methods, Issues \& Ethics. New York, Peter Lang, 2004, pp. 1-12.

DUARTE, R. Práticas Comunicacionais e Sociais dos Cosplayers no Brasil: uma Análise dos Processos Online e Offline através do Orkut. Dissertação de mestrado (Comunicação e Linguagens). Curitiba, Programa de Pós-graduação em Comunicação e Linguagens da Universidade Tuiuti do Paraná, 2008.

ELM, M. "How do Various Notions of Privacy Influence Decisions in Qualitative Internet Research?", in A. Markham; N. Baym. Internet Inquiry. Conversations About Method. Los Angeles, Sage, 2009. ESCOBAR, A. "Welcome to Cyberia. Notes on the Anthropology of Cyberculture", in Current Anthropology, v. 35, n. 3, June/1994, pp. 211-31. Disponível em: http://www.unc.edu/ aescobar/text/ eng/arturowelc.pdf. Acesso: 25/1/2009.

ESS, C. Digital Media Ethics. Cambridge, Polity Press, 2009.

GUTIERREZ, S."AEtnografia Virtual na Pesquisa de Abordagem Dialética em Redes Sociais On-line", in 32a ReuniãoAnualdaAnped.Caxambu.Sociedade, CulturaeEducação:Novas Regulações?Rio de Janeiro, Associação Nacional de Pós-Graduação e Pesquisa em Educação, 2009. Disponível em: http:// www.anped.org.br/reunioes/32ra/arquivos/trabalhos/GT16-5768--Int.pdf . Acesso: 25/12/09. HAKKEN, D.Cyborgs @ Cyberspace?:An Ethnographer Looks Into the Future. New York, Routledge, 1999. HARAWAY, D. Simians, Cyborgs and Women: The Reinvention of Nature. New York, Routledge, 1991. HINE, C. Virtual Ethnography. London, Sage, 2000. (ed.). Virtual Methods. Oxford, Berg, 2005.

."How Can Qualitative Internet Researchers Define the Boundaries of Their Project?", in A. Markham; N. Baym. Internet Inquiry. Conversations About Method. Los Angeles, Sage, 2009, pp. 1-20. HODKINSON, P. "Insider Research' in the Study of Youth Cultures", in Journal of Youth Studies, v. 18, 2005, pp.131-49.

JANKOWSKI, N.; VAN SELM, M. “Epilogue: Methodological Concerns and Innovations in Internet Research", in C. Hine (ed.). Virtual Methods. Oxford, Berg, 2005, pp. 199-208.

JENKINS, H. Fans, Bloggers and Gamers. Exploring Participatory Culture. New York, New York University Press, 2006.

JOHNS,M.D.;CHEN, S.;HALL, G.OnlineSocial Research.Methods, Issues\&Ethics. NewYork, PeterLang, 2004. JONES, S. (ed). Doing Internet Research. Critical Issues and Methods for Examining the Net. London, Sage, 1999.

KENDALL, L. “How Can Qualitative Internet Researchers Define the Boundaries of Their Project? A Response to Christine Hine", in A. Markham; N. Baym. Internet Inquiry. Conversations About Method. Los Angeles, Sage, 2009, pp.21-5.

KOZINETS, R. V. Netnography: Doing Ethnographic Research Online. London, Sage, 2010. MARKHAM, Annette. Life Online: Researching Real Experience in Virtual Space. Walnut Creek, Altamira Press, 1998.

MARKHAM, A.; BAYM, N. Internet Inquiry. Conversations About Method. Los Angeles, Sage, 2009. 
MITSUISHI, Y. "Entre Graphos e Ethos: uma Abordagem Crítica a Etnografia Virtual", in J. Ribeiro; S. Bairon (orgs.). Antropologia Visual e Hipermídia. Lisboa, Afrontamento, 2007. Disponível em: http://realidadesintetica.com/pdfs/mitsuishi_ymgraphosethos.pdf. Acesso: 5/11/2009.

MONTARDO, S. "Redes Temáticas na Web e Biossocialidade On-line", in GT Comunicação e Cibercultura do XVIII Encontro da Compós - Associação Nacional dos Programas de Pós-graduação em Comunicação. Belo Horizonte PUCMG, junho de 2009. Disponível em: http://www.compos. org.br/data/biblioteca_1022.pdf. Acesso: 5/11/2009.

; ROCHA, P.J. “Netnografia: Incursões Metodológicas na Cibercultura”, in Revista E-compós, Revista Nacional dos Programas de Pós-graduação em Comunicação, vol. 4. Brasília, dezembro de 2005. Disponível em: http://www.compos.org.br/seer/index.php/e-compos/article/viewFile/55/55. Acesso: 2/8/2007.

NATAL, G. Comunicação e Construção de Perfis de Consumo e Identidades na Internet: a Marca Mary Jane. Dissertação de mestrado (Comunicação e Linguagens). Curitiba, Programa de Pós-graduação em Comunicação e Linguagens da Universidade Tuiuti do Paraná, 2009.

ORGADI, S. "How Can Researchers Make Sense of the Issues Involved in Collecting and Interpreting Online and Offline Data?", in A. Markham; N. Baym. Internet Inquiry. Conversations About Method. Los Angeles, Sage, 2009, pp. 33-53.

RHEINGOLD, Howard. The Virtual Community: Surfing the Internet. Cambridge, MIT Press, 1993.

RUBY, J. "¿Son los Medios Interactivos Una Alternativa a los Filmes Etnográficos?", in Enl@ce: Revista Venezolana de Información, Tecnología y Conocimiento, 6 (3), dez./2009, pp. 81-93. Disponível em: http://revistas.luz.edu.ve/index.php/enlace/article/viewFile/3257/3143. Acesso: 15/2/2010.

RYAN, J. The Virtual Campfire: an Ethnography of Online Social Networking. Dissertação de mestrado. Dissertação (Antropologia). Connecticut, Wesleyan University, 2008. Disponível em: http:// www.thevirtualcampfire.org/virtualcampfire.htm. Acesso: 5/1/2010.

SÁ, S. "Netnografias nas Redes Digitais", in J. L. Prado Crítica das Práticas Midiáticas. São Paulo, Hacker, 2002.

STERNE, J. "Thinking the Internet: Cultural Studies Versus the Millennium", inJ. Steve (ed). Doing Internet Research. Critical Issues and Methods for Examining the Net. London, Sage, 1999, pp. 257-83.

STONE, A. R. "Will the Real Body Please Stand Up? Boundaries Stories About Virtual Cultures", in M. Benedikt (ed). Cyberspace: First Steps. Cambridge, MIT Press, 1991.

STRANGELOVE, M. "Virtual Video Ethnography: Towards a New Field of Internet Cultural Studies", in Revista Interin, n. 3, junho de 2007. Disponível em: http://www.utp. br/interin/artigos/art_livre_01_strangelove.pdf. Acesso: 20/6/2007

TURKLE, S. Life on the Screen: Identity in the Age of the Internet. New York, Touchstone, 1997.

TURNER, F. From Counterculture to Cyberculture. Stewart Brand, the Whole Earth Network and the rise of the Digital Utopianism. Chicago, The University of Chicago Press, 2006.

VIANA, L. Música na Cibercultura: Reconfiguração da Estrutura do Mercado a partir da Desintermediação do Funk Brasileiro e sua Produção em Rede. Dissertação de mestrado (Comunicação e Linguagens). Curitiba, Universidade Tuiuti do Paraná, 2009.

WARD, M. "Thoughts on Bloggin as an Ethnographic Tool", in Annual Ascilite Conference: Who's Learning? Whose Technology?, 23. Sydney, The University of Sydney, 2006, pp. 843-51. Disponível em: http://www.ascilite.org.au/conferences/sydney06/proceeding/pdf_papers/p164.pdf . Acesso: 18/1/2008.

WILLIAMS, R. Television and Social Form. London, Fontana, 1974.

WINKIN, Y. A Nova Comunicação. Da Teoria ao Trabalho de Campo. Campinas, Papirus, 1998. 